\title{
Image Features Based Intelligent Apple Disease Prediction System: Machine Learning Based Apple Disease Prediction System
}

\author{
Mahvish Jan, Central University of Jammu, Jammu, India \\ (iD) https://orcid.org/0000-0002-1327-521X \\ Hazik Ahmad, Sher-e-Kashmir University of Agricultural Sciences and Technology of Kashmir, India
}

\begin{abstract}
A pattern classifier (PC) is used to solve a variety of non-separable and complex computing problems. One of the key problems is to efficiently predict a type of disease in a typical fruit tree. The timely and accurately predicted disease in an apple tree may help a farmer to take appropriate preventive measures in advance. In this article, an apple disease diagnosis system is developed to predict the apple scab and leaf/spot blight diseases. In this article, low level and shape-based features are used for the development of an intelligent apple disease prediction system. First, the key image features like entropy, energy, inverse difference moment (IDM), mean, standard deviation (SD), perimeter, etc., are extracted from the apple leaf images. The model for the proposed system is trained by using multi-layer perceptron (MLP) pattern classifier and eleven apple leaves image features. The Gradient descent back-propagation algorithm is used for building the intelligent system to carry out the pattern classification. The proposed system is tested using some random samples and exhibits excellent diagnosis accuracy of $99.1 \%$. The sensitivity of the proposed prediction model is $98.1 \%$ and specificity of $\sim 99.9 \%$.
\end{abstract}

\section{KEYWORDS}

Alternaria Leaf Blight, Apple Scab, Fruit Diseases, Image Features, Intelligent Systems, Multi-Layer Perceptron, Pattern Classifier, Supervised Learning

\section{INTRODUCTION}

Kashmir is famous for its specialty of apples all over India. India contributes $65 \%$ of total production of apples in the world. Like other fruits, apple (Malus pumila) is also attacked by a number of diseases such as apple scab and Alternaria leaf spot/blight. These diseases can affect both the leaves and fruit which may amount loss in both the quality as well as quantity (Kayalvizhi and Antony, 2011). Apple Scab disease is caused by the fungus Venturia inaequalis. It infects leaves, shoots, buds, blossoms and fruit. It is the most serious and prevalent disease. At the initial stage, the symptoms usually appear 
as small spots on the lower side of leaves or as spots on either surface of older leaves. The disease causes pale yellow or brown or olive-green color on the upper surface of the leaves. The lesions on older leaves are more definite in outline and become velvety grey to sooty black. The lesion may form a convex surface with corresponding convex area on the lower side. If infection is severe, leaves will become twisted, distorted and stunted and tend to fall prematurely. Alternaria leaf spot/blight is caused by a fungal pathogen Alternaria Mali. The lesions of this disease in apple first appears on leaves in late spring and early summer as round, small, purplish or blackish spots. Initially the leaf spots are $1 / 8$ to $1 / 4$ in diameter. The disease spreads rapidly over a temperature between 77 to $86^{\circ} \mathrm{F}$ $\left(25\right.$ to $\left.30^{\circ} \mathrm{C}\right)$ and due to wet conditions. At ideal temperatures infection occurs with 5.5 hours of wetting, and lesions can appear in the orchard two days after infection causing a serious epidemic. The main objective of this research work is to predict the apple disease by using the low-level image features of the leaves of an apple tree. The texture or statistical features of leaves of a healthy tree and other one with diseases are distinctive which may be used to train a machine leaning model. The automatic prediction of diseases in apple trees in the Kashmir valley of India has been addressed by this research work. The contribution of the work is that it achieves better prediction accuracy for apple disease by using only low-level texture or shape features of apple leaf images. The system mainly focuses with a new dataset of apple leaf images collected afresh from Kashmir region of India where $75 \%$ of the total apples are grown in India.

The rest of the paper is organized as follows. The section two of the paper provides a brief background about machine learning based solutions for disease prediction in various fruits and vegetables. The third section of the article provides the detailed architecture and algorithm used for our proposed apple disease prediction system. The next section describes about the experimental setup and results analysis for the proposed system. The last section provides a comparison of the proposed technique with the existing similar techniques with brief conclusion.

\section{BACKGROUND}

In recent years, different methodologies have been discussed by various authors in detection of plant diseases. Pooja Pawar et al. (2016) extracted the texture features for detection of cucumber disease and then provided necessary treatment for the disease using feed forward back propagation network as a classifier (Pawar, 2016). S. Patil et al. (2015) have proposed a methodology of detecting grape disease by using colour features and back propagation algorithm for classification (Patil, 2015). P. Mohanaiah et al. (2013) in the paper (Mohanaiah et al., 2013) have proposed a method of how to obtain the statistical texture features by using Grey Level Co-occurrence Matrix (GLCM). As GLCM'S are very useful to large number of data, the number of grey levels is often reduced (Mohanaiah et al., 2013). Bhavini J. Samajpati et al. (2016) have used random forest classifier for classifying the apple diseases on fruit namely apple scab, apple rot and apple blotch. The authors have extracted texture features and colour features of the apple images and have combined them using feature level fusion. For segmentation k-means clustering algorithm is used. For extracting the colour features they have used Grey colour histogram and colour coherence vector. Local binary pattern, Complete local binary pattern, Local ternary pattern is used for texture feature extraction (Samajpati and Degadwala, 2016). Zhang Jian et al. (2010) in the paper (Zhang et al., 2010) have used SVM classifier for recognition of cucumber disease. The three features have been extracted from the image namely colour, texture and shape from spot image. Downy mildew, Brown spot, Angular leaf spot have been classified using different kernel functions. The authors have computed the accuracy of different kernel function and it is concluded that SVM identification method with RBF kernel function gives more accurate results (Zhang et al., 2010). Jobin Francis et al. (2016) have proposed a method to classify pepper leaf spot disease. In this, at first, RGB image is converted into HSV colour space as hue and saturation contains most of the information. The image segmentation is done on the basis of Masking process and threshold is used for image segmentation. In the masking process, 1's and 0's were assigned 
to the unhealthy and healthy regions of the leaf respectively. If the intensity of the green pixels is greater than the predefined value its assigned 0 value and after masking these pixels were discarded. The leaf mask and the damaged mask has been used for analysis. The diseased portion is detected using damaged ratio (DR). Useful Features are extracted from GLCM approach and passed through the classifier (Francis et al., 2016). Sima Kumari et al. (2015) have extracted shape and roundness of apple fruit and have used neural network for classification (Kumari et al., 2015). A. Camargo et al. (2008) have taken images of five different crops and have used image processing techniques for disease detection (Camargo et al., 2008). Arti N. Rathod et al. (2014) has used image processing and feed forward neural networks for detection of leaf disease (Rathod et.al 2014). Sandesh Raut et al. (2017) have detected disease by using SVM classifier (Raut et al., 2017). Nivedita. Kakade et al. (2015) have proposed system for the detection of grape disease by using texture features (Kakade et al. 2015). Zhang et al. (2010) have proposed a system for detection of citrus canker disease (Zhang et al., 2010). Sanjeev S. Sannakki et al. (2013) have proposed methodology for detection of grape leaf diseases (Sannakki et al., 2013). Sachin D. Khirade et al. (2015) have proposed a prediction system for detection of leaf disease by using feed forward network as a classifier (Khirade et al., 2015). Abdul Kadir et al. (2011) have proposed a system using probabilistic neural network as a classifier by extracting colour, vein, shape, and texture features of leaves (Kadir et al., 2011).

The literature survey highlights that most of the fruit diseases has been diagnosed by using random forest classifier, SURF algorithm for pattern classification. Furthermore, most of the techniques are based on the parameters like colour, texture, size and shape of the leaf of a tree or a plant. The major focus of the research has been restricted to cucumber disease, cotton, sugarcane, etc. Bhavini et al. have developed a prediction system using k-means clustering and random forest classifier where authors have not computed the diagnostic accuracy of the system. Moreover, another structure proposed by Meinanda et al. (2015) for apple disease diagnosis where they have calculated performance of $85 \%$ which is comparatively low. Therefore, the further research on apple disease prediction system may focus on using pattern classifier like multi-layer perceptron (MLP) by using low level features of leaf of apple disease system. However, deep level features may be used to train the proposed model for apple disease, but this work will mainly focuses on some low level features of apple leaves for intelligent prediction of the apple diseases. The presented research survey also reveals that most research have focused mainly on machine leaning based solutions rather than deep learning for disease prediction in plants and trees like apple, cucumber etc.

\section{PROPOSED APPLE DISEASE PREDICTION SYSTEM}

\section{Framework for Proposed System}

The framework of apple disease prediction system is given in Figure 1. The objective of the system is to develop a prediction system for apple disease. The various steps involved in proposed framework are discussed below.

The various images of apple leaf disease have been collected from the various regions of Kashmir. These images are used to train the proposed neural network. One of the Images shown in Figure 2 depicts (a)Apple scab and (b) Alternaria leaf spot/blight.

\section{Image Preprocessing}

In this step, the acquired RGB format images are converted into the grayscale images. None of the image filtering techniques has been used as the quality of the acquired images was good.

\section{Feature Extraction}

Feature Extraction is used to extract the pertinent features from an image and represent that information in a lower dimensionality space (Aggarwal and Aggarwal, 2012). Texture refers to how the gray levels 
Figure 1. The framework for the proposed apple disease prediction system Image acquisition

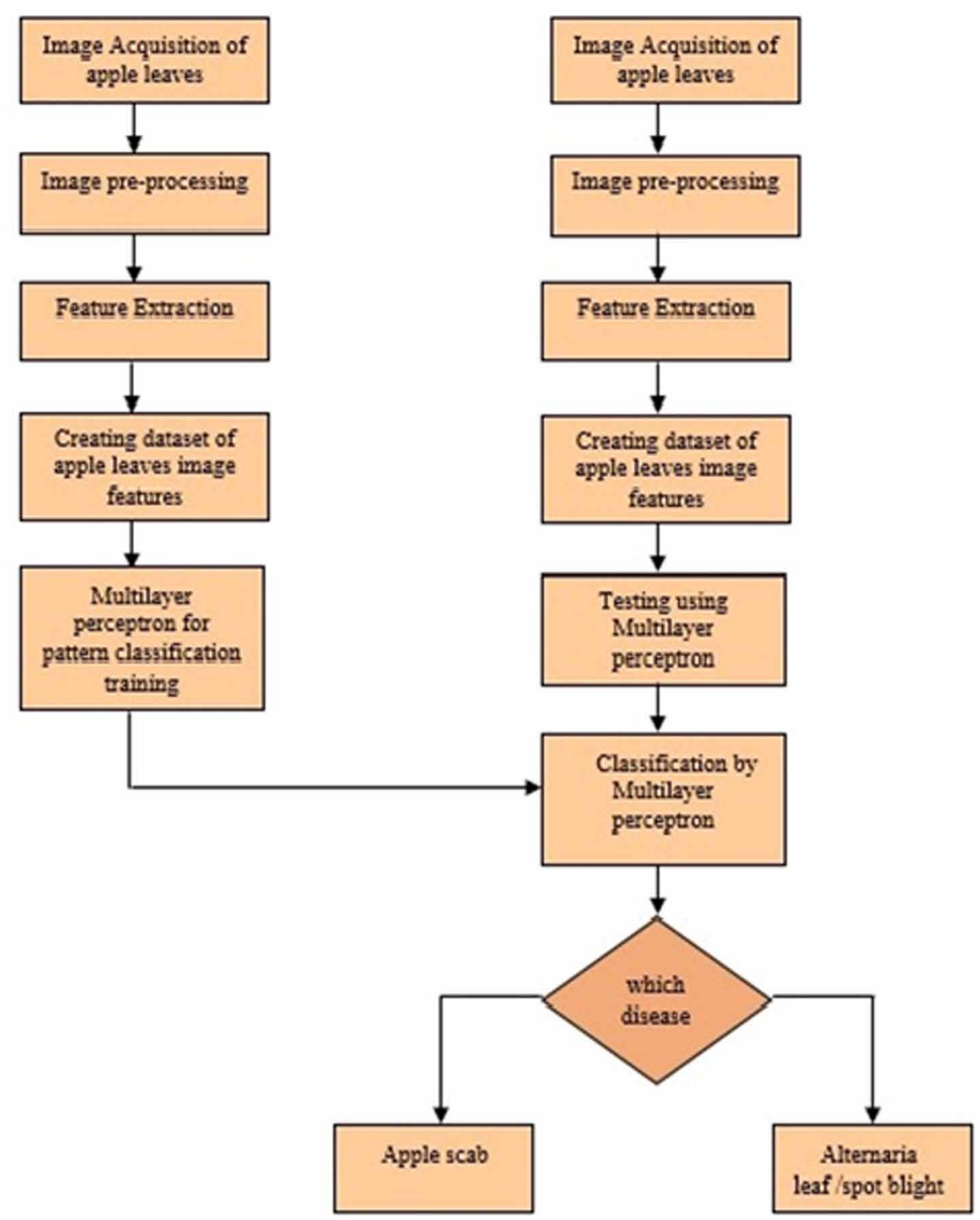

of an image are distributed over the pixels. The motive of this step is to extract the features from an image. These features will be used to determine apple diseases. Many features can be extracted from an image such as color features, texture features, boundary and edge detection, shape features. Texture features can be extracted from various methods such as statistical, structural, model based, and transformation information. Out of these image features, texture and shape features are extracted from an image and are used for training the neural network. This is done by using first order statistical and second order statistical methods. First order statistical methods include mean, kurtosis, standard deviation, skewness while second order statistical moments include Gray Level Co-occurrence Matrix (GLCM) features. 
Figure 2. Image preprocessing: (a) Original image; (b) Grayscale image

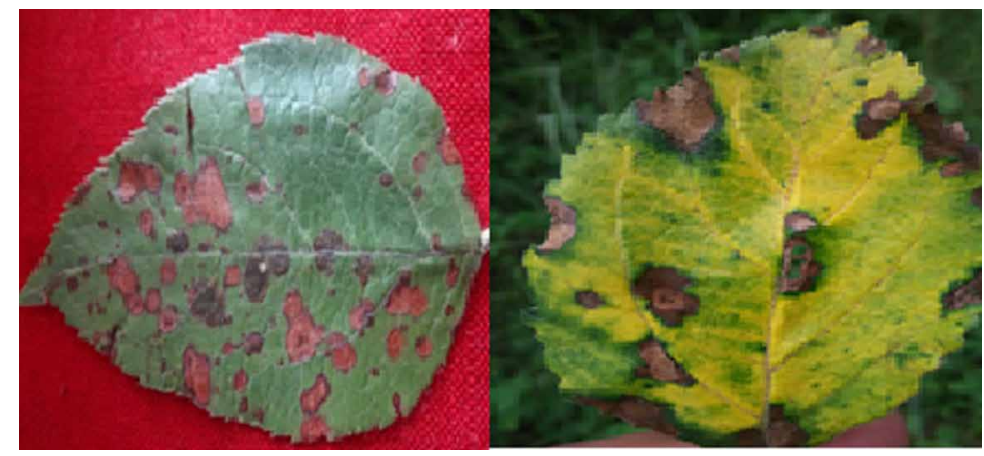

Figure 3. Image preprocessing: (a) Original image; (b) Grayscale image
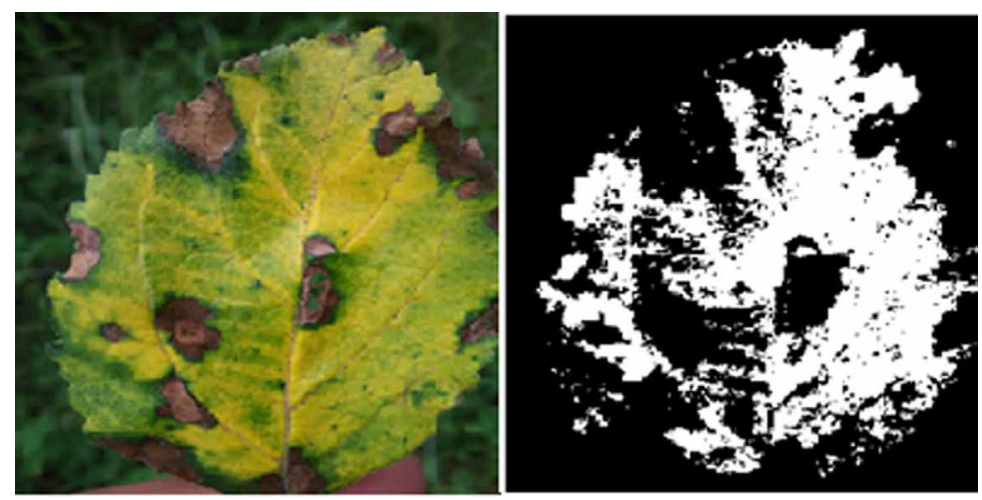

\section{First Order Statistical Moments}

The first order statistics determines the properties of an image region by exploiting the spatial interactions between the gray level distributions of an image. Various first order statistical moments are discussed below:

- Mean: Mean refers to average value of intensity of an image. It is denoted by $\mu$ and is given in Equation 1:

$\mu=\frac{1}{N} \Sigma_{I=1}^{N} X_{i}$

where $\mathrm{N}$ is the total no of pixels present in the image, xi represents value at the $i^{\text {th }}$ pixel.

- Kurtosis: Kurtosis is the measure of uniformity. It determines whether the data are heavy-tailed or light-tailed relative to the normal distribution. It is given in Equation 2:

kurtosis $=\mathrm{E}\left(\frac{Z-\mu}{\sigma}\right)^{4}$ 
where $\mu$ is the mean, $\sigma$ is the standard deviation.

- Standard deviation: It is mostly used method to measure variability or dispersion in statistics. In terms of image processing, it measures how far pixel values of an image vary from the mean. A low value of standard deviation indicates data points are close to mean and a high value of standard deviation indicates that the data points are spread out over a wide range of values. It is denoted by $\sigma$ and is given in Equation 3:

$\sigma=\sqrt{\frac{1}{N}} \sum_{i=1}^{N}(X i-\mu)^{2}$

where $\mu$ is the mean, $\mathrm{N}$ is the no of pixels, $\mathrm{xi}$ represents value at the ith pixel.

- Skewness: Skewness is the measure of degree of symmetry of dataset or distribution. Symmetric data should have skewness near to zero. In symmetrical distribution the value of skewness zero. Skewness is related to third order moment. Data can be positively skewed or negatively skewed. Positively skewed means that result of computation is greater than zero (e.g. darker and glossier regions), whereas negatively skewed means that result of computation is less than zero (e.g. lighter and dull surfaces). It is given $n$ Equation 4:

Skewness $=E\left[\left(\frac{z-\mu}{\sigma}\right)^{3}\right]$

where $\sigma$ is the standard deviation and $\mu$ is the mean.

\section{Second Order Statistical Moments (GLCM)}

The GLCM is a way of extracting second order statistical texture features. It is used to measure the variation of intensity of the pixel of interest. A GLCM is a matrix containing no of rows and columns, where no of rows and columns is equal to no of gray levels ' $G$ '. It is used to measure the relative frequency between the gray levels of neighborhood pixel. Suppose $\mathrm{T}(i, j, \Delta m, \Delta n)$ the relative frequency separated by a pixel distance $(\Delta m, \Delta n)$ occur within a given neighborhood, one with intensity ' $i$ ' and other with intensity ' $\mathrm{j}$ '. Gray level Co-occurrence matrix is computed based on two parameters displacement and orientation. For classification, five GLCM texture features are extracted which are used for training of neural network in the next step. It takes into account how often a pixel with gray value come either vertically, horizontally, and diagonally to the neighborhood pixels with value $\mathrm{j}$. It is quantized in four directions: horizontal $\left(0^{\circ}\right)$, vertical $\left(90^{\circ}\right)$ and diagonally, bottom left to top right $\left(-45^{\circ}\right)$ and top left to bottom right $\left(-135^{\circ}\right)$.

- Contrast: Contrast is the difference in the brightness and color that makes an object distinct with other object in the same field of view. Its value is zero for constant image. It is defined in the Equation (5) as:

contrast $=\sum_{i=0}^{G-1} \sum_{j=0}^{G-1}(i-j)^{2} T(i, j)$ 
Figure 4. Gray level co-occurrence matrix (GLCM)
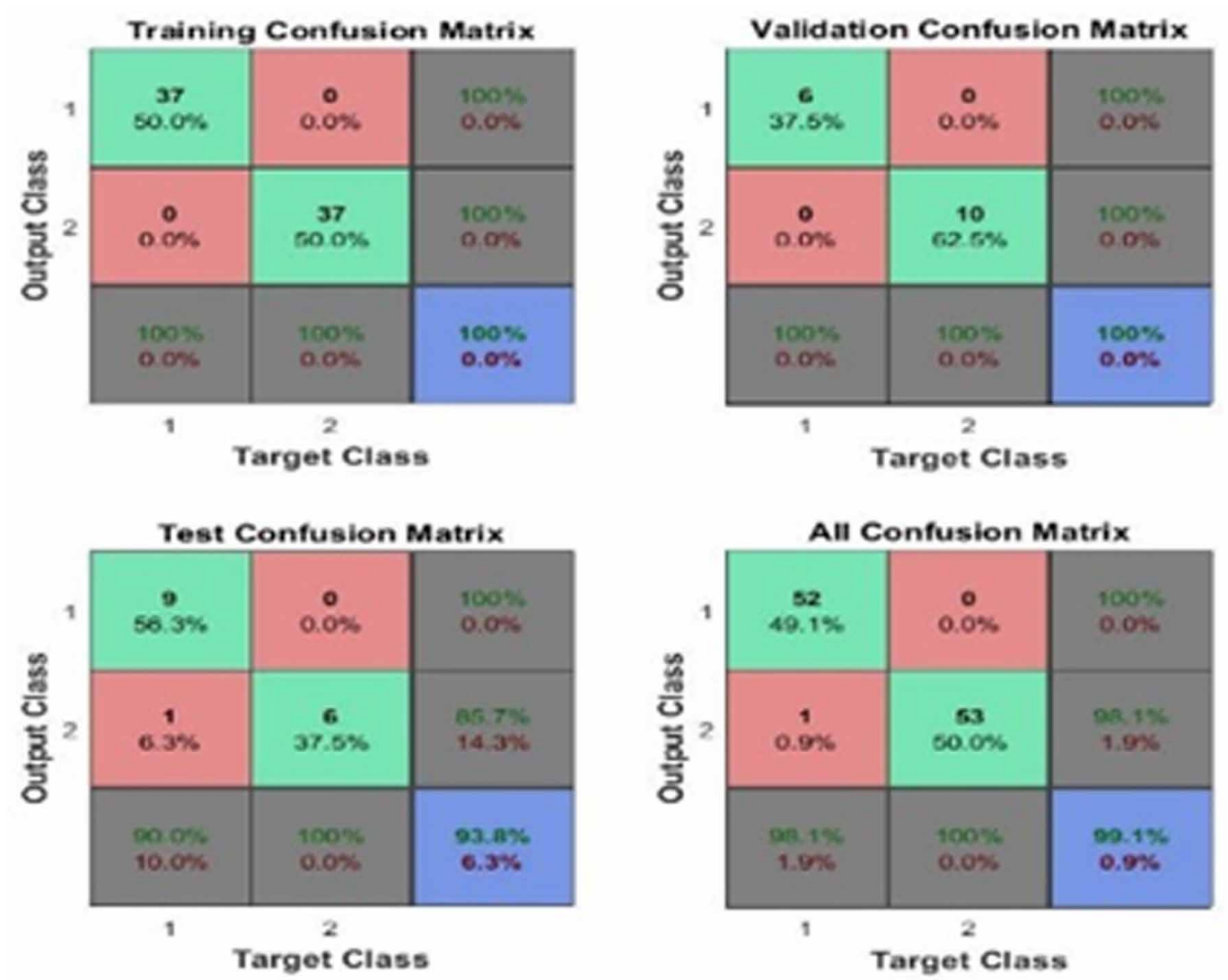

- Correlation: It is a measure of how a pixel is correlated to its neighbor over the whole image. Its range is between -1 and 1 . Its value is $\mathrm{NaN}$ for constant image. The correlation can be also defined by the below Equation 6:

Correlation $=\sum_{i=0}^{G-1} \sum_{j=0}^{G-1} \frac{(i-\mu)(j-\mu) T(i, j)}{\sigma_{i} \sigma_{j}}$

where $\sigma$ is the standard deviation, $T(i, j)$ represents relative frequency of that pixel,. $\mu$ is the mean.

- Entropy: Entropy is a measure of uncertainty or randomness that can be used to characterize the texture of the input image. When all the elements of grey level co-occurrence matrix are same the value of entropy will be maximum. It is also defined in the Equation 7 given below:

Entropy $=\sum_{i=0}^{G-1} \sum_{j=0}^{G-1}(T(i, j) * \log (T(i, j))$

where $(i, j)$ represents position of the pixel of an image and $T(i, j)$ represents relative frequency of that pixel. 
- Inverse difference moment (IDM): Inverse Difference Moment (IDM) is also used to measure the texture of an image. It is also known as Entropy. It measures homogeneity of an image. Its value will be maximum when most of the occurrences in GLCM are concentrated near the main diagonal. It is inversely proportional to GLCM contrast. Equation 8 defines Inverse difference moment:

$\mathrm{IDM}=\sum_{i=0}^{G-1} \sum_{j=0}^{G-1} \frac{T(i, j)}{1+(i, j)^{2}}$

where $(i, j)$ represents position of the pixel of an image and $T(i, j)$ represents relative frequency of that pixel.

- Energy: It's also known as angular second moment. It measures the pixel pair repetitions. It measures the uniformity of an image. Value of energy is 1 for constant image. It is also defined as shown in Equation 9 below:

Correlation $=\sum_{i=0}^{G-1} \sum_{j=0}^{G-1}\left[(T(i, j)]^{2}\right.$

where $(i, j)$ represents position of the pixel of an image and $T(i, j)$ represents relative frequency of that pixel.

\section{Shape Features}

Shape is one of the most important features for disease detection. Many features can be extracted from the leaf such as perimeter, area, eccentricity of the leaf, etc. One of the shape features can be used for apple disease detection. In this proposed framework, perimeter of the leaf has been used for apple disease detection.

\section{The Algorithm for Proposed System}

The pseudo code for the proposed algorithm is shown in Figure 5. Initially, the apple leaf image is read and preprocessed to obtain the gray scale image with improved quality. The resultant is used to extract the unique features present in the apple leaf by using an inbuilt function like gray co-matrix and region-props. In this manner steps 1-3 are repeated for a mixed category of 106 apple leaves to build a dataset. Pattern classifier is chosen and trained with the help of multilayer perceptron. Finally, performance of the proposed system is computed by using confusion matrix.

\section{Pattern Classifier for the Proposed System}

There are many classifiers used for classification of objects depends on the type of learning. There are two main types of classification used to classify output: Supervised classification and unsupervised classification. Unsupervised classification methods comprise of: k-means clustering, adaptive resonance theory, self-organizing map, etc. However, supervised classification methods contain: multilayer perceptron, decision tree algorithm, etc. Proposed methodology uses Multi-Layer Perceptron for classification. Multi-Layer Perceptron is mostly used feed forward neural network, which produces set of outputs corresponding to a set of inputs. It is called workhorse of learning in neural networks. It is an example of supervised learning Feed forward network consists of input layer, one or more hidden layers and an output layer and these layers are allied to each other by a weighted connection. Feed forward means that data flows only in one path from input nodes through hidden nodes (if any) 


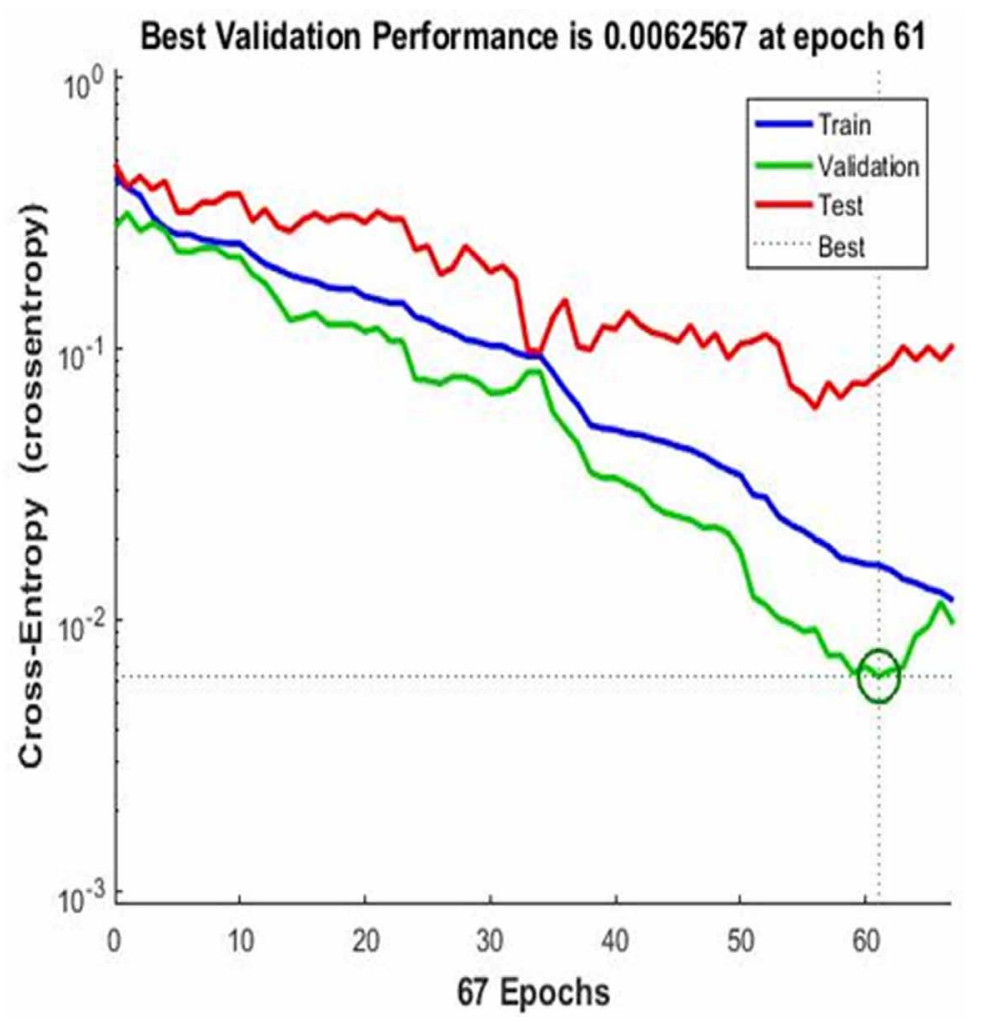

to output nodes. Each node, has a non-linear activation function (usually sigmoid) excluding input nodes. The multilayer perceptron (MLP) is shown in Figure 6. It consists of an input layer consisting of three nodes. The bias node has value equal to 1 and the other two nodes are having external inputs as M1 and M2. The outputs from nodes in the output layer are 1, M1, M2 are fed to the hidden layer because no computation is made in the input layer. Inputs of the hidden layer will be the output of input layer. Output of the hidden layer can be computed by applying activation function. The outputs are then fed to the output layer as an input. After applying activation function to the input of output layer, it produces $\mathrm{O} 1$ and $\mathrm{O} 2$ as output. $\mathrm{f}$ is the activation function, wi is the sum of the weights and $\mathrm{xi}$ is the total input. Multilayer perceptron learns by a process called backpropagation algorithm. It is a supervised scheme for training that means the network learns from the labeled data. The aim of back propagation algorithm is to optimize the weights.

\section{BACK PROPAGATION ALGORITHM}

This section describes about the famous back-propagation algorithm for training of typical multilayer perceptron.

1. Forward pass:

a. Weight Initialization 


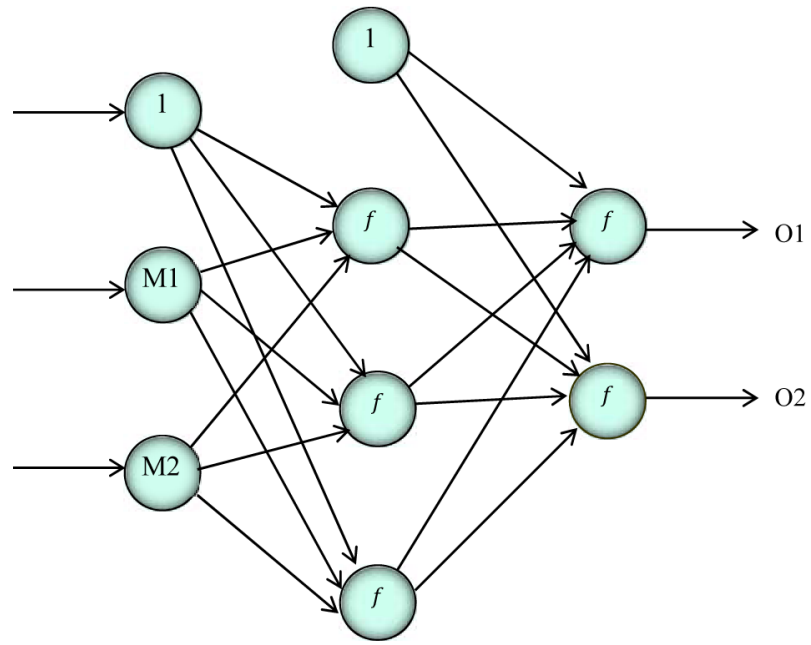

\section{Input Layer Hidden Layer Output Layer}

Initially the weights are assigned randomly. For each input during the training the ANN is activated and the output is observed. This output is compared with the target output. If the observed output is not equal to the target output the error is propagated back to the previous layers:

$\mathrm{xi}=\mathrm{X} 1+\mathrm{X} 2+\mathrm{X} 3+\ldots$

$\mathrm{wi}=\mathrm{w} 1+\mathrm{w} 2+\mathrm{w} 3+\ldots$

$y$ in $=\sum_{i=1}^{n} w i x i$

Apply activation function "f" (sigmoid function) to net input, to get net output:

Yout $=\mathrm{f}($ Yin $)$

$\mathrm{f}=\frac{1}{1+\mathrm{e}^{-y_{\text {in }}}}$

b. Error calculation

Error is calculated by Least Mean-square error method. E(tot)is the error calculated as:

Etot $=\sum 1 / 2(\text { target }- \text { actual })^{2}$ 


\section{Backward pass}

The calculated error is given back to the hidden layer. The goal of backward pass is to minimize the error. The error is calculated by gradient descent method by taking the partial derivative of the total error with respect to weight that is wi at hidden layer. This can be calculated as shown in Equation 14:

$$
\left(\frac{\partial E_{t o t}}{\partial w_{i}}\right)=\left(\frac{\partial E_{t o t}}{\partial y o u t_{k}}\right) *\left(\frac{\partial \text { yout }_{k}}{\partial \text { yin }_{k}}\right) *\left(\frac{\partial y i n_{k}}{\partial w_{i}}\right)
$$

\section{EXPERIMENTAL SETUP}

For experimentation, the dataset for the proposed apple disease prediction system has been captured by using a high definition camera with resolution 350dpi. The dataset consists of 106 instances of apple leaf images comprising of Apple scab disease, Alternaria leaf blight and healthy leaves. As shown in the Table, out of the 106 instances 35 leaves belongs to Apple Scab disease, 36 belongs to Alternaria leaf blight and remaining instances belongs to healthy tree. Majority of the leaf images has been captured in .jpg or .png format. The apple leaf images have been captured from apple trees in the various parts of Kashmir region of India.

\section{Performance Parameters}

In this section, performance evaluation parameters are used to evaluate the performance. For experiment various types of images of apple leaves are used. In order to carry out the study we have taken into consideration two types of apple diseases namely Apple scab, Alternaria leaf blight. In literature survey, various performance measures have been proposed for evaluation of learning models. Among all, the commonly used performance parameters are:1) Sensitivity 2) Accuracy and 3) Specificity.

- Sensitivity: Sensitivity of a prediction system may be defined as the ratio of actual positives which are predicted positive to the sum of actual positives and false negatives. This can be written as shown in Equation 15:

Sensitivity $=\frac{T P}{T P+F N}$

- Specificity: Specificity of a prediction system may be defined as the proportion of correctly predicted negatives to the sum of actual true negatives and false positives. This can be written as shown in Equation 16:

Specificity $=\frac{T N}{T P+F N}$

- Accuracy: Accuracy of a prediction system may be defined as the proportion of correctly predicted observation to the total number of observations. It is shown in the Equation 17:

Accuracy $=\frac{T N+T P}{T P+T N+F N+F P}$ 
Table 1. Apple dataset used for experimentation

\begin{tabular}{|l|l|l|l|}
\hline \multicolumn{1}{|c|}{ Sr. No. } & \multicolumn{1}{c|}{ Type of Leaf } & Number of Instances. & \multicolumn{1}{c|}{ Dpi of Images } \\
\hline 1 & Apple scab disease & 35 & 350 \\
\hline 2 & Alternaria leaf blight & 36 & 350 \\
\hline 3 & Healthy & 35 & 350 \\
\hline
\end{tabular}

where TP denotes true positive, FN denotes false negative, FP denotes false positive, TN denotes true negative.

In a more general way, Sensitivity is how well a model identifies positive cases whereas Specificity means how well a model identifies negative cases, Accuracy is how well it identifies both positive as well as negative cases. The measure of accuracy is dependent on sensitivity as well as accuracy. If the value of sensitivity and specificity is high(low), the value of ac-curacy will be high(low). In terms of prediction system, high value of sensitivity means to correctly identify diseased leaf, high value of specificity means to correctly identify those with-out disease. Table 2 shows the confusion matrix.

Table 2a. Confusion matrix

\begin{tabular}{|l|l|l|l|}
\hline & \multicolumn{1}{|c|}{ Disease } & \multicolumn{1}{c|}{ Non-Disease } & \multicolumn{1}{c|}{ Total } \\
\hline Positive & A(True Positive) & B(False Positive $)$ & TTest Positive \\
\hline Negative & C(False Negative) & D(True Negative) & TTest Negative \\
\hline & Tdisease & Tnon-diseased & Total \\
\hline
\end{tabular}

Table $2 \mathrm{~b}$. Testing and validation accuracy of proposed system

\begin{tabular}{|l|l|l|l|l|l|l|}
\hline $\begin{array}{c}\text { No. of } \\
\text { Training } \\
\text { Instances }\end{array}$ & $\begin{array}{c}\text { No. of } \\
\text { Iterations }\end{array}$ & $\begin{array}{c}\text { Error } \\
\text { Gradient }\end{array}$ & $\begin{array}{c}\text { Positive } \\
\text { Predicted } \\
\text { Value (\%) }\end{array}$ & $\begin{array}{c}\text { Negative } \\
\text { Predicted } \\
\text { Value (\%) }\end{array}$ & $\begin{array}{c}\text { Validation } \\
\text { Accuracy }\end{array}$ & $\begin{array}{c}\text { Testing } \\
\text { Accuracy }\end{array}$ \\
\hline 20 & 41 & $6.77 \mathrm{e}-07$ & 100 & 100 & 100 & 100 \\
\hline 40 & 37 & 0.0210 & 95.2 & 100 & 100 & 83.3 \\
\hline 60 & 36 & 0.0310 & 92.6 & 94.2 & 87.5 & 87.5 \\
\hline 80 & 31 & 0.0217 & 95.1 & 97.4 & 100 & 100 \\
\hline 106 & 46 & 0.0351 & 100 & 96.4 & 93.8 & 93.8 \\
\hline
\end{tabular}

\section{PERFORMANCE ANALYSIS}

The various performance analysis parameters are shown in the Table 2 and Table 3 . The testing accuracy indicates the overall prediction of the proposed system where the data set was split into training and testing segments. This prediction accuracy is used to compute the performance of the proposed system with some instances selected randomly from the same dataset. Whereas in the case of validation accuracy, the proposed system is evaluated for performance by using new instances other than the dataset used for training purpose. This type of accuracy is generally used to evaluate the performance of the system in new environment by using some randomly selected samples from other population than the one used for training of the model. 
Table 3. Performance measures of proposed system

\begin{tabular}{|l|l|l|l|l|}
\hline $\begin{array}{c}\text { No. of Training } \\
\text { Instances }\end{array}$ & No. of Iterations & \multicolumn{1}{|c|}{ Sensitivity (\%) } & Specificity (\%) & \multicolumn{1}{|c|}{$\begin{array}{c}\text { Overall Accuracy } \\
(\%)\end{array}$} \\
\hline 20 & 41 & 100 & 100 & 100 \\
\hline 40 & 37 & 100 & 95.0 & 97.5 \\
\hline 60 & 36 & 94.3 & 92.5 & 93.4 \\
\hline 80 & 31 & 97.5 & 95 & 96.3 \\
\hline 106 & 46 & 96.2 & 100 & 99.1 \\
\hline
\end{tabular}

The number of iterations required for training the proposed model is not much dependent upon the size of dataset. It is also observed that the positive predicted value is higher for lower size of samples whereas it lies between $95.2 \%$ to $92.6 \%$ for the sample size 40 to 80 . Negative predicted values are higher for lower size samples it lies in between $97.4 \%$ to $96.4 \%$. Validation accuracy for a large size of dataset is $93.8 \%$ and for small size of dataset it is $100 \%$.

The Table 3 shows the performance of proposed system with respect to number of training instances and overall accuracy. The system exhibits excellent results using 106 samples with overall accuracy of $99.1 \%$, sensitivity of $96.2 \%$ and specificity of $100 \%$. However, the number of iterations required has a poor correlation with the number of training instances.

The Figure 7 shows the classification accuracy using confusion matrix by taking 106 instances of apple disease leaf features namely Apple scab and leaf spot/blight. The overall accuracy of the system is $99.1 \%$. The green color in the confusion matrix indicates correctly identified instances of apple disease. The red color indicates incorrectly identified instances. Grey color indicates positive predicted value and negative predicted value. Blue color in the confusion matrix indicates overall performance

Figure 8 shows the performance plot indicating cross entropy curve where the goal is to minimize the number of misclassified training samples. The blue line indicates decreasing error on training data. Green line indicates validation error, training stops when validation error stops decreasing. Red line shows error on test data indicating how well network will generalize training data. A ROC plot shown in Figure 9 indicates the percentage of true positive predictions we get as a function of how many false positive classifications are accepted.

\section{COMPARATIVE ANALYSIS}

The Table 4 illustrates the performance comparison of the proposed apple disease prediction system with the similar types of prediction systems. The proposed system exhibits an overall accuracy of 99.1\% which is much higher in comparison to the similar system developed by pooja Pawar et al. (2016) for cucumber disease with accuracy of $80.4 \%$ and Meinda et al. (2015) for apple disease with an accuracy of $85 \%$. The proposed system uses more image features like correlation, contrast, entropy, mean, standard deviation, etc.

The proposed system results in high prediction accuracy as compared to the other similar approaches used by researchers. The proposed system has an advantage that it only utilize the low level texture and shape image features of apple leaf trees for building the intelligent apple prediction system which further outperform its counterparts in terms of prediction accuracy of about $99 \%$. The proposed intelligent apple disease prediction system may be effectively used by developing full scale application software with proper Graphical User Interface (GUI) for providing the input images with a handheld scanner. Moreover, the captured apple leaf image may be captured and then fed to the system through GUI to predict the class of disease or health of the tree. 
International Journal of Agricultural and Environmental Information Systems Volume 11 • Issue 3 • July-September 2020

Figure 7. Confusion matrix
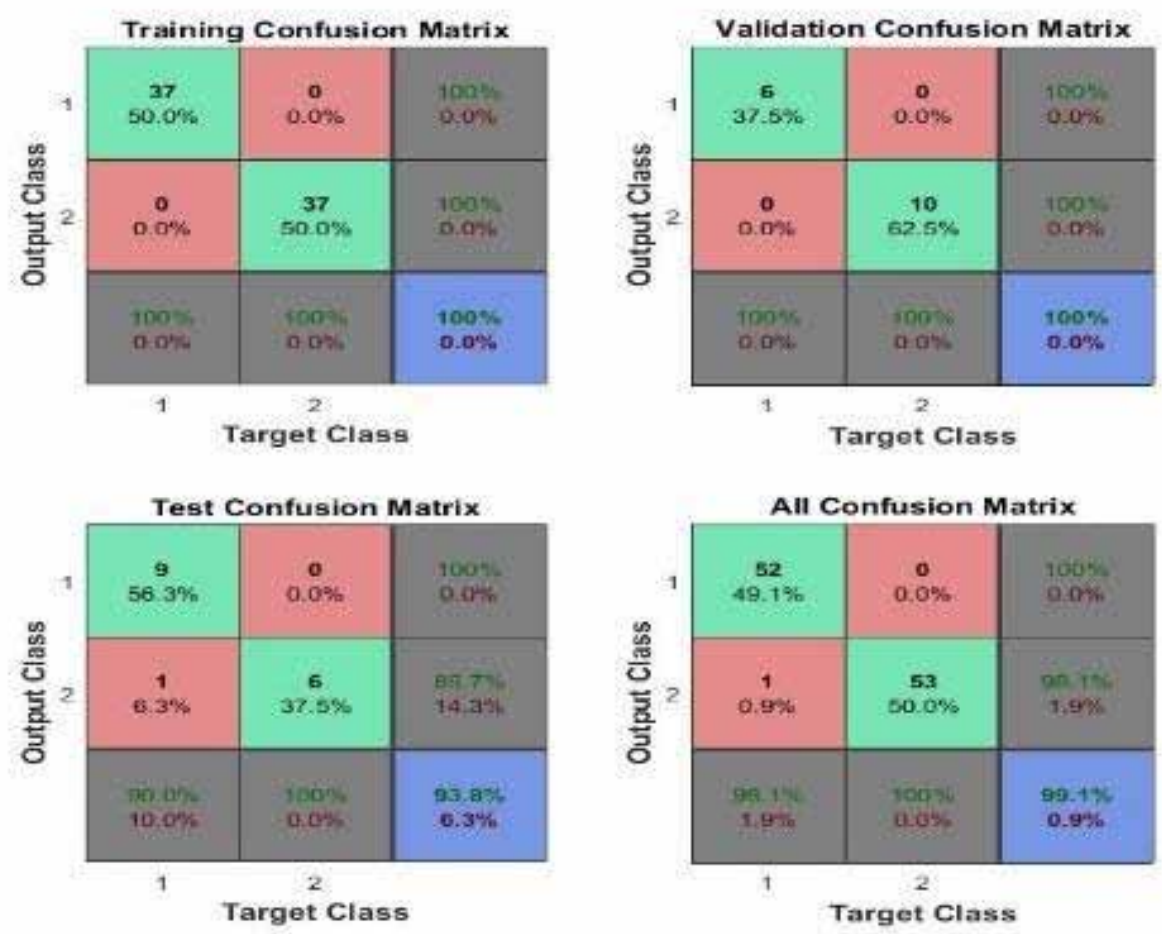

Figure 8. Validation results after training

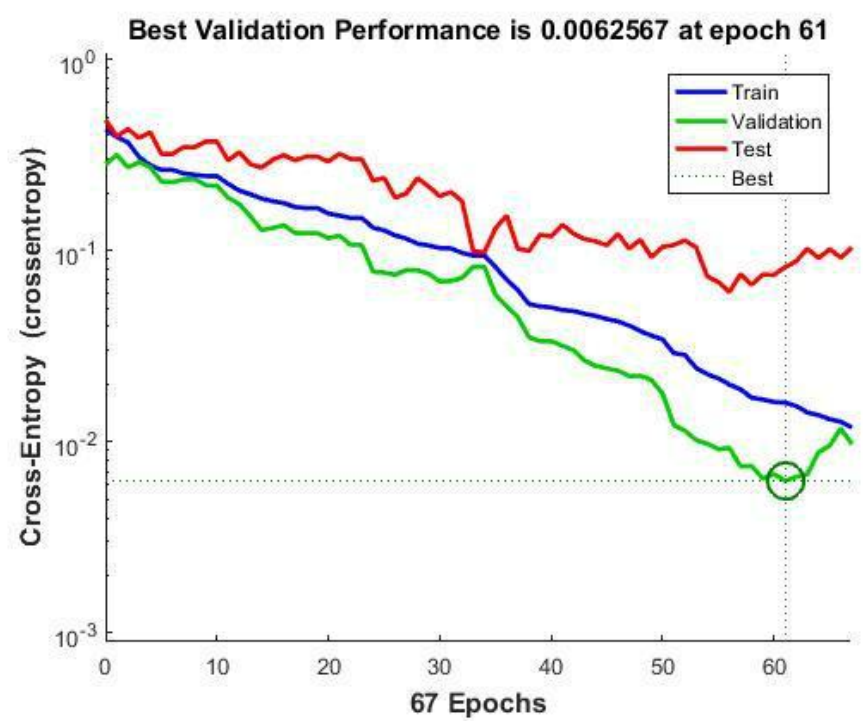


Figure 9. Receiver operator characteristic plot
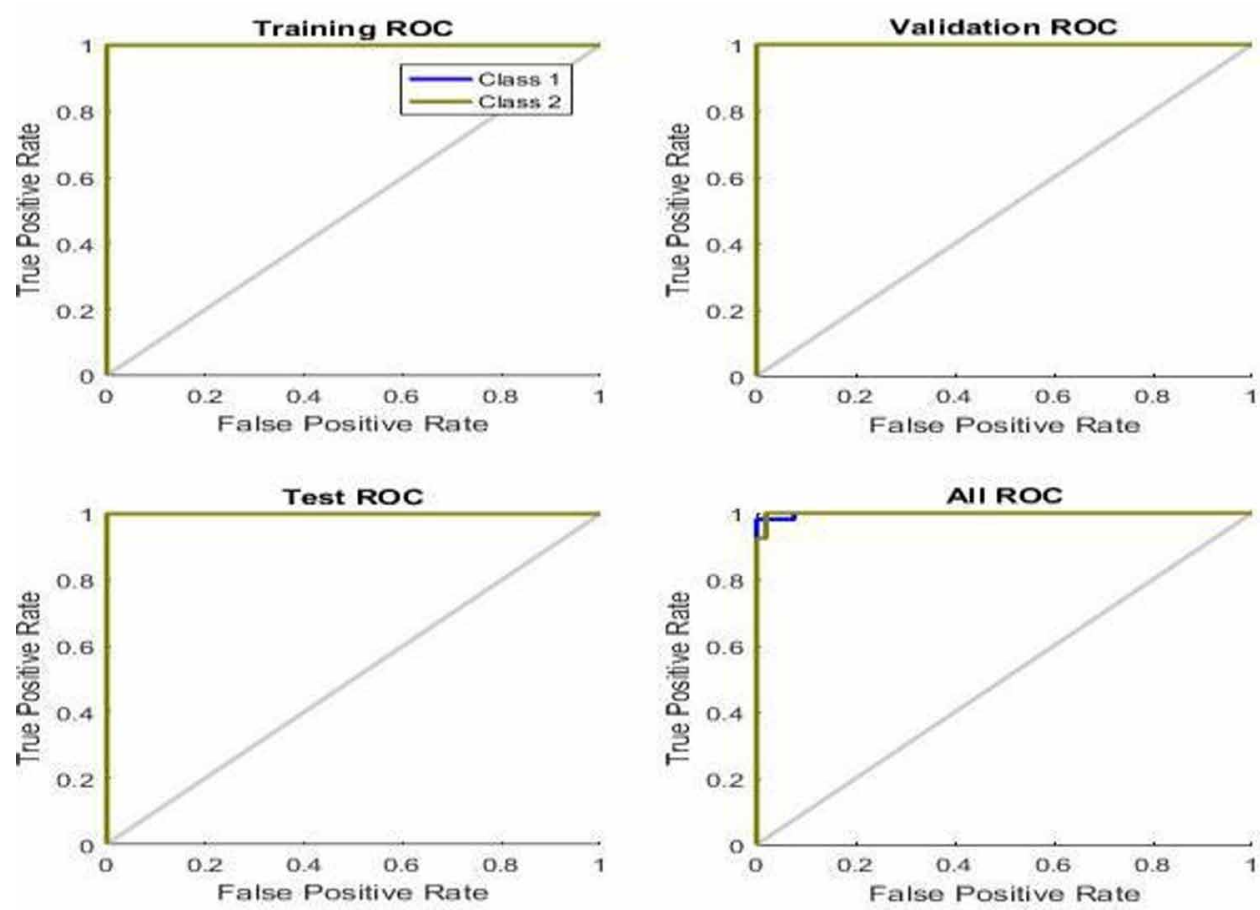

Table 4. Comparative analysis

\begin{tabular}{|l|l|l|l|l|}
\hline \multicolumn{1}{|c|}{ Disease Name } & \multicolumn{1}{c|}{ Network Used } & \multicolumn{1}{c|}{ Author } & \multicolumn{1}{c|}{ Year } & Performance \\
\hline Cucumber Disease & $\begin{array}{l}\text { Feed Forward Back- } \\
\text { Propagation Network }\end{array}$ & $\begin{array}{l}\text { Pooja Pawar, Dr. } \\
\text { Varsha Turkar, Prof. } \\
\text { Pravin Patil }\end{array}$ & 2016 & $80.4 \%$ \\
\hline Apple Disease & $\begin{array}{l}\text { K-Means algorithm, } \\
\text { SURF algorithm }\end{array}$ & $\begin{array}{l}\text { Bashir Sajo Mienda, } \\
\text { Adibah Yahya, } \\
\text { Ibrahim A. Galadima, } \\
\text { Mohd Shahir Shamsir }\end{array}$ & 2015 & $85 \%$ \\
\hline Apple Disease & $\begin{array}{l}\text { Gradient Descent } \\
\text { with Back- } \\
\text { propagation network }\end{array}$ & $\begin{array}{l}\text { Mahvish Jan, Arvind } \\
\text { Selwal }\end{array}$ & 2018 & $99.1 \%$ \\
\hline
\end{tabular}

\section{CONCLUSION AND FUTURE SCOPE}

The research work presented in this paper has been carried out to diagnose the diseases of apple fruits. The research focused on two popular types of diseases namely apple scab and leaf/spot blight. The GLCM and first order statistical moments have been used to extract texture features. The perimeter and texture features of the leaf have been used for training the neural network. The proposed system provides classification accuracy of $99.1 \%$. In future, more apple leaf features may be considered with hybrid classifier to achieve better results. As a future scope, the Deep learning neural networks like Convocational Neural Networks (CNN) may be used for training the model which will skip the 
feature engineering step for effective implementation of the system. Support vector machine (SVM) may be used to build the similar model for apple disease diagnosis. Moreover, wireless sensors may be deployed in orchards for real life diagnosis of apple diseases.

\section{ACKNOWLEDGMENT}

We are very thankful to Central University of Jammu for providing all the amenities for carrying out the work. 


\section{REFERENCES}

Aggrwal, N., \& Aggrwal, R. K. (2012). First and Second Order Statistics Features for Classification of Magnetic Resonance Brain Images. J. Signal Inf. Process., 3(2), 146-153.

Camargo, A., \& Smith, J. S. (2009). An image processing based algorithm to automatically identify plant disease visual symptoms. Biosystems Engineering, 102(1), 9-21J. doi:10.1016/j.biosystemseng.2008.09.030

Francis, J., \& Anoop, B. K. (2016, March). Identification of leaf diseases in pepper plants using soft computing techniques. In Proceedings of the 2016 conference on emerging devices and smart systems (ICEDSS) (pp. 168173). IEEE.

Kadir, A., Nugroho, L. E., Susanto, A., \& Santosa, P. I. (2013). Leaf classification using shape, color, and texture features.

Kayalvizhi, V., \& Antony, U. (2011). Microbial and physico-chemical changes in tomato juice subjected to pulsed electric field treatment. African Journal of Agricultural Research, 6(30), 6348-6353S.

Khirade, S. D., \& Patil, A. B. (2015, February). Plant disease detection using image processing. In Proceedings of the 2015 International conference on computing communication control and automation (pp. 768-771). IEEE.

Kumar, G., \& Bhatia, P. K. (2014, February). A detailed review of feature extraction in image processing systems. In Proceedings of the 2014 Fourth international conference on advanced computing \& communication technologies (pp. 5-12). IEEE.

Mienda, B., Yahya, A., Galadima, I. A., \& Shamsir, M. S. (2015). Analysis of Apple Fruit Diseases using Neural Network. Research Journal of Pharmaceutical, Biological and Chemical Sciences, 5(388), 388-396.

Mohanaiah, P., Sathyanarayana, P., \& GuruKumar, L. (2013). Image texture feature extraction using GLCM approach. International journal of scientific and research publications, 3(5), 1-5.

Patil, S. P., Kumbhar, V. P., Yadav, D. R., \& Ukirade, N. S. (2015). Detection of leaf diseases by image processing. IJARECE, 4(4), 909-913.

Pawar, P., Turkar, V., \& Patil, P. (2016, August). Cucumber disease detection using artificial neural network. In Proceedings of the 2016 International Conference on Inventive Computation Technologies (ICICT) (Vol. 3, pp. 1-5). IEEE.

Rathod, A. N., Tanawala, B. A., \& Shah, V. H. (2014). Leaf disease detection using image processing and neural network. [IJAERD]. International Journal of Advance Engineering and Research Development, 1(6).

Raut, S., \& Fulsunge, A. (2017). Plant disease detection in image processing using Matlab. International journal of innovative Research in science. Engineering and Technology, 6(6), 10373-10381.

Samajpati, B. J., \& Degadwala, S. D. (2016, April). Hybrid approach for apple fruit diseases detection and classification using random forest classifier. In Proceedings of the2016 International Conference on Communication and Signal Processing (ICCSP) (pp. 1015-1019). IEEE.

Zhang, J., \& Zhang, W. (2010). Support vector machine for recognition of cucumber leaf diseases. In Proceedings of the 2nd IEEE International Conference on Advanced Computer Control ICACC 2010 (Vol. 5, pp. 264-266). IEEE Press; . doi:10.1109/ICACC.2010.5487242

Zhang, M., \& Meng, Q. (2010, December). Citrus canker detection based on leaf images analysis. In Proceedings of the $2^{\text {nd }}$ international conference on information science and engineering (pp. 3584-3587). IEEE; . doi:10.1109/ ICISE.2010.5691630 